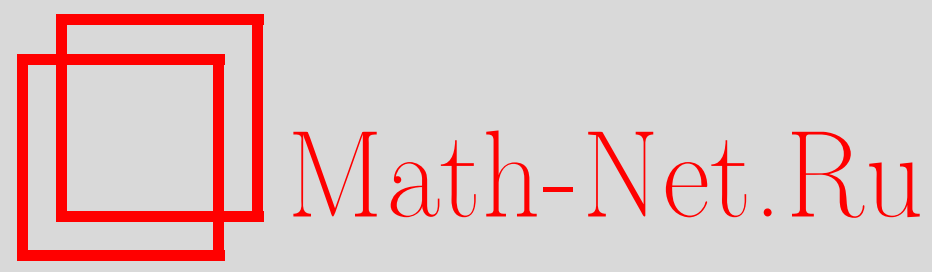

А. Я. Белов, Линейные рекуррентные уравнения на дереве, Матем. заметки, 2005, том 78, выпуск 5, 643-651

DOI: https://doi.org/10.4213/mzm2628

Использование Общероссийского математического портала Math-Net.Ru подразумевает, что вы прочитали и согласны с пользовательским соглашением http://www . mathnet.ru/rus/agreement

Параметры загрузки:

IP: 107.22 .136 .117

26 апреля 2023 г., 13:51:06

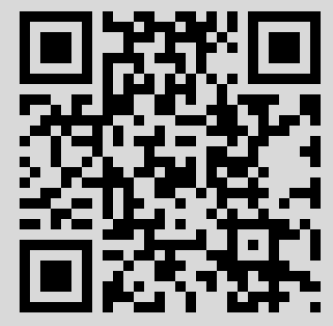




\title{
ЛИНЕЙНЫЕ РЕКУРРЕНТНЫЕ УРАВНЕНИЯ НА ДЕРЕВЕ
}

\author{
А. Я. Белов
}

Построен алгоритм проверки существования нетривиального решения системы линейных рекуррент на дереве, а также его нахождения.

Библиография: 8 названий.

Известно, что проблема равенства в конечно определенной полугрупше, а следовательно, и алгебре, алгоритмически неразрешима. С другой стороны, эта проблема разрешима, если идеал соотношений задан конечным базисом Грёбнера, замкнутым относительно композищии [1]. В этой связи В.Н. Латьшев в 1980 году поставил вопрос о существовании алгоритма определения, является ли данный элемент делителем нуля или нильпотентом в случае, когда идеал соотношений задан конечным базисом Грёбнера. Предполагаемьй ответ на этот вопрос отрицателен, поэтому Латышевым был поставлен также аналогичньй вопрос для мономиальных автоматных алгебр. Для этого случая существование алгоритма проверки, является ли данньй элемент нильпотентом, было установлено В.В. Борисенко, а существование алгоритма для делителей нуля В.В. Борисенко и А.Я. Беловым [2]. Аналогичньй результат для некоторого класса квадратичных алгебр был получен Н. К. Иыуду [3], [4], а также Д. И. Пионтковским [5].

В свое время автором была предложена (но не опубликована) идея так назьваемых алгебр с ограниченной переработкой (по аналогии с теорией малых сокращений в группах), варианты которых затем рассматривались в [3], [4], [6]. Ряд результатов получил обобщение для этого класса алгебр. Оно вытекает из свойств линейных рекуррент на деревьях. Путь доказательства, основанный на идеях динамического программирования, позволяет показать алгоритмическую разрешимость проблемы распознавания делителей нуля и сделать ряд других результатов прозрачными. Понятие алгебр с ограниченной переработкой позднее стало в различных вариациях использоваться и другими авторами.

Пусть $A$ - алгебра над полем $\mathbb{F}$ с фиксированным набором образующих $a_{1}, \ldots, a_{s}$. Словом в алгебре называется слово от ее образующих, $|W|$ обозначает длину слова $W$. Порядку на образующих $a_{1} \prec a_{2} \prec \cdots \prec a_{s}$ отвечает лексикографический порядок на множестве слов. Пусть $\prec-$ некоторое отношение линейного порядка на множестве слов такое, что убьвающие цепи обрьваются. Тогда слова, не представимые в виде линейных комбинаций меньших слов, образуют нормальный базис алгебры $A$ и называются нормальными словами (относительно данного порядка). Алгебра назьвается мономиальной, если определяющие соотношения имеют вид $\left\{w_{i}=0\right\}_{i \in I}, w_{i}$ - некоторые 
слова. Мономиальная алгебра назьвается автоматной (см. [2], [7]), если существует конечный ориентированный граф Г (возможно, с петлями и мультиребрами), каждое ребро которого помечено буквой некоторого конечного алфавита $\mathscr{A}$. Множество определяюших соотношений в $A$ получается путем приравнивания нулю слов, которые нельзя прочитать вдоль пути по графу $Г$. Алгебра $A$ однозначно определяется по Г и потому задается конечным числом данных. Известно [2], [7], что любая автоматная алгебра задается детерминированным графом (т.е. у которого из каждой вершины выходит не более одной стрелки, помеченной данной буквой) с единственной начальной вершиной. При этом существует алгоритм построения такого графа по произвольному [2], [7], и только такие графы в дальнейшем рассматриваются.

ОПРЕДЕЛЕНИЕ. Размеченным ориентированным деревом $T$ называется следующий объект.

1. Ориентированное дерево с начальной вершиной $O$ такое, что все ребра, исходящие из каждой вершины, помечены разными буквами, принадлежащими некоторому конечному алфавиту $\mathscr{A}$.

2. В начальную вершину $O$ стрелки не входят, в любую другую вершину входит ровно одна стрелка. Каждой вершине $v$ отвечает поддерево $T_{v}$ дерева $T$, образованное вершиной $v$ и всеми нижележащими вершинами (дерево считается растущим вниз, так что $O$ есть верхняя вершина).

3. Все вершины разбиты на конечное число типов, которое будет обозначаться $m(T)$. Тип вершины вместе с меткой исходящей стрелки однозначно определяет тип следуюшей вершины (т.е. однозначно определяются типы соседних нижележащих вершин).

4. Если $v_{1}$ и $v_{2}-$ вершины одного типа, то существует канонический изоморфизм $\varphi_{v_{1} v_{2}}: T_{v_{1}} \rightarrow T_{v_{2}}$ поддеревьев $T_{v_{1}}$ и $T_{v_{2}}$, который переводит вершину (ребро) в вершину (ребро) того же типа.

ЗАмечАниЕ. Множество путей в конечном детерминированном автомате с единственной начальной вершиной (а следовательно, и множество ненулевых слов в автоматной мономиальной алгебре) естественным образом образует структуру размеченного ориентированного дерева.

ОПРЕДЕЛЕНИЕ. Системой линейных рекуррент $C$ на размеченном дереве $T$ (или СЛР) назьвается следуюший объект.

1. Каждой вершине $v=v_{i}$ дерева $T$ соответствует конечномерное векторное пространство $V=V_{i}$ над $F$ и некоторая векторозначная переменная $\xi=\xi_{i}$ со значениями в $V_{i}$.

2. Каждой вершине $v$ дерева $T$ соответствует конечный набор линейных уравнений на переменные, отвечающие $v$, а также некоторым нижележащим вершинам. Эти уравнения имеют вид

$$
\sum_{i} f_{i}^{(v)}\left(\xi_{i}\right)=0
$$

где $f_{i}^{(v)} \in V_{i}^{*}, \xi_{i} \in V_{i}$, пространства $V_{i}$ отвечают некоторым вершинам, лежашим ниже вершины $v$, причем таких вершин конечное число. Иньми словами, 
каждой вершине соответствует конечное множество конечных наборов коэффициентов (линейных функционалов). Следующее условие означает, что это соответствие зависит только от типа вершины.

3 . Если $v_{1}$ и $v_{2}$ суть две вершины одного типа, то с каноническим изоморфизмом $\varphi_{v_{1} v_{2}}: T_{v_{1}} \rightarrow T_{v_{2}}$ связан канонический изоморфизм соответствуюших векторных пространств, а также наборов уравнений.

Если $T$ - луч и $\operatorname{dim}\left(V_{i}\right)=1$ для всех $i$, то СЛР есть система линейных рекуррент так, как их обычно понимают (см. [8]).

Отметим, что в каждое уравнение входит только конечное число переменных. Из конечности числа типов вершин и конечности числа переменных, участвующих в каждом уравнении, вытекает

ПРЕДЛОЖЕНИЕ 1. Для любой системы линейных уравнений на дереве Т существует натуральное $n$ такое, что в любом уравнении, соответствующем произвольной вершине $v$, не участвуют переменные, соответствующие вериинам, находящимся от $v$ на расстоянии, бо́льшем $n$.

Минимальное такое $n$ называется степенью системы линейных рекуррент на $T$ и обозначается $n=\operatorname{deg}(C)$. Решением системы $C$ называется такой набор значений переменных, удовлетворяющих системе $C$, что почти все (т.е. все, кроме конечного числа) эти значения равны нулю. Решение называется тривиальным, если оно нулевое, и нетривиальным в противном случае. Фрагментом дерева $T$ при вершине $v$ называется поддерево дерева $T_{v}$, составленное из вершин, лежащих на расстоянии не вьше $n$ от вершины $v$ и расположенных не выше. Пусть $\left\{V_{i}\right\}$ есть набор пространств, отвечающих вершинам фрагмента, и $\forall i \xi_{i} \in V_{i}$. Тогда набор векторов $\left\{\xi_{i}\right\}$ называется вектором фрагмента.

Размерность $r(C)$ пространства векторов фрагмента, отвечаюших СЛР $C$, не превосходит произведения $d(C)$ - максимальной размерности пространства, отвечающего вершине дерева $T,-$ и максимального числа вершин во фрагменте, и ограничена при фиксированном $T$. Ясно, что $r(C) \leqslant b(T)^{n}$, где $n=\operatorname{deg}(C), b(T)$ есть максимальное число стрелок, исходящих из вершины дерева.

Наша цель - доказать следующую теорему.

ТЕОРемА 1. Существует алгоритм проверки существования нетривиального решения системы линейных рекуррент на дереве, а также нахождения какого-нибудь такого решения.

Ясно, что каждая СЛР задается конечным набором данных. В самом деле, если считать эквивалентньми вершины с одинаковыми метками, то фактор-граф дерева $T$ по такому отношению эквивалентности - конечньй ориентированньй графф с отмеченной начальной вершиной (граф Уфнаровского), по которому $T$ однозначно восстанавливается. Все маршруты длины не вьше $n$, исходяшие из данной вершины графа Уфнаровского, образуют конечное дерево, и все фрагменты дерева - в точности такие деревья. Таким образом, линейные пространства достаточно задавать на конечном множестве вершин графа Уфнаровского, а уравнения - на конечном множестве фрагментов.

Данная теорема вытекает из вспомогательных утверждений и конструкций, которые будут приведены ниже. 
ОПРЕДЕЛЕНИЕ. Вектор фрагмента назьвается решаемым, если он продолжается до решения СЛР на поддереве $T_{v}$, и $l$-решаемым, если он продолжается до решения СЛР на поддереве $T_{v}$, для которого значения всех переменных, отвечающих вершинам, находящимся на расстоянии ниже $l$ от вершины $v$, нулевые.

Ясно, что решаемый вектор является $l$-решаемьм при некотором натуральном $l$. Ясно также, что $l$-решаемые векторы образуют векторное пространство, которое мы обозначим $L_{v, l}$. Для вершин $v$ и $v^{\prime}$ одинакового типа эти пространства канонически изоморфны и мы их, когда это не приведет к недоразумениям, будем отождествлять.

Сделаем несколько несложных наблюдений.

ЛЕмма 1. При фиксированном $l$ существует алгоритм вычисления базиса в пространстве $L_{v, l}$.

ДокАЗАТЕльСтво. Если все векторы, отвечающие вершинам, находящимся на расстоянии большем, чем $l$ от вершины $v$, считать равными нулю, то в уравнениях, связанных с поддеревом $T_{v}$, будет участвовать только конечное число переменных и число уравнений будет конечно. Очевидно, что в этом случае есть алгоритм проверки наличия нетривиального решения.

ЛЕмма 2. Имеем $L_{v, l+1} \supseteq L_{v, l}, \operatorname{dim}\left(L_{v, l+1}\right) \geqslant \operatorname{dim}\left(L_{v, l}\right)$.

Поскольку пространство $L_{v, l}$ есть подпространство пространства векторов фрагмента, размерности пространств $L_{v, l}$ равномерно ограничены (причем по всем типам сразу). Поэтому при некотором $l$ наступит стабилизация $L_{v, l+1}=L_{v, l}$, причем для всех типов сразу.

ЛЕмма 3. Пусть при некотором $l>n=\operatorname{deg}(C)$ для всех типов вериин выполняется равенство $L_{v, l+1}=L_{v, l}$. Тогда $L_{v, l+2}=L_{v, l+1}$ и при всех $k \in \mathbb{N}$ $L_{v, l+k}=L_{v, l}$.

ДокАзАтЕльство. Каждому дереву $T_{v}$ отвечает набор поддеревьев, связанных с вершинами $\left\{v_{i}\right\}$, соседними снизу, и каждому $(l+1)$-решаемому фрагменту дерева $T_{v}$ отвечает согласованньй набор $l$-решаемых фрагментов для соответствующих поддеревьев. Верно и обратное. Поскольку $l>n$, каждое из решений, соответствующих этим поддеревьям, можно заменить на решение, у которого переменные, отвечающие вершинам, лежащим на расстоянии вьше $l$ от корневой, принимают нулевые значения. Поэтому любой $(l+1)$-решаемый фрагмент является также $(l+2)$-решаемьм.

Теперь существование алгоритма, требуемого в теореме, и его построение становится ясно в силу приведенных лемм. Основная теорема доказана.

Из доказательства основной теоремы следует также оценка на число $l$, для которого наступает стабилизация. Это число оценивается величиной $r(C) d(C)$.

К СЛР на деревьях сводятся многие алгоритмические задачи. Рассмотрим дерево, отвечающее ненулевьм мономам автоматной (в частном случае, конечно определенной) мономиальной алгебры. Они образуют некоторое размеченное дерево T. При этом вершина, отвечающая моному $u_{1}$, соединена с вершиной, отвечающей моному $u_{2}$, стрелкой, на которой написана буква $a_{i} \Longleftrightarrow u_{2}=u_{1} a_{i}$. Если мономиальная алгебра $A$ автоматна, то каждому моному $M$ отвечает вершина конечного графа Уфнаровского (т.е. конец пути, по которому его можно прочитать, стартуя от начальной вершины). Тем самым 
получается разметка множества ненулевых мономов в конечное число цветов, а значит и вершин дерева $T$.

Векторноепространство, отвечающеепроизвольной вершине, одномерно и совпадает с основным полем $\mathbb{F}$. Элементу $x$ алгебры $A$ отвечает конечная линейная комбинация слов или набор коэффициентов при соответствующих словах, причем только конечное их число - не нули.

Пусть элемент $f \in A$ фиксирован. Через $\lambda_{f}\left(M_{1}, M_{2}\right)$ обозначим коэффициент, с которым входит моном $M_{1}$ в произведение $M_{2} f ; \delta\left(M_{2}, x\right)$ - коэффициент, с которьг входит моном $M_{2}$ в элемент $x \in A$. Равенству $x f=0$ в алгебре $A$ отвечает СЛР (зависящая от $f$ ), которая получается путем приравнивания коэффищиентов:

$$
\sum_{M \in T} \lambda_{f}(N, M) \delta(M, x)=0, \quad N \in T .
$$

Если $M, N$ находятся на расстоянии $>d=\operatorname{deg}(f)$, то $\lambda_{f}(N, M)=0$. Кроме того, $\lambda_{f}(N, M)$ зависит только от типа вершины $N$ и пути, соединяющего $M$ и $N$. Ясно, что условия теоремы 1 вьполняются и мы имеем

СлЕДСТВИЕ 1 (из основной теоремы). Для данной мономиальной автоматной алгебры А существуют алгоритмы проверки следующих утвержсдений:

а) данный әлемент $x \in A$ (т.е. линейная комбинация слов) является делителем нуля;

б) данный әлемент $g \in A$ принадлежит левому (соответственно, правому) идеалу, заданному конечным набором әлементов;

в) алгоритмически разрешим также вопрос о том, является ли идеал свободным левым (правым) А-модулем с данной конечной системой порохсдающих.

ЗАмЕчАниЕ. Заметим, что аналогичного алгоритма проверки принадлежности элемента двустороннему идеалу не существует, ибо проблема равенства нулю элемента в произвольной алгебре (а также полугрупе), заданной конечным набором образующих и определяющих соотношений, алгоритмически неразрешима даже для свободной алгебры.

ДокАЗАТЕЛЬСтво. Достаточно прояснить вопрос о том, как выписать СЛР, отвечающую данной алгоритмической задаче в алгебре. Пункт а) получается из конструкции, приведенной перед формулировкой данного предложения (кроме того, он является частным случаем п.в)).

В пш. б) и в) достаточно ограничиться случаем правых идеалов. Вершинам дерева, как и раньше, отвечают слова. В п. б) надо проверить разрешимость уравнения

$$
\sum_{i=1}^{k} x_{i} f_{i}=g
$$

где $\left\{f_{i}\right\}_{i=1}^{k}$ - образуюшие левого идеала $L ; g \in A$ - элемент, принадлежность которого идеалу $L$ проверяется; $x_{i}$ суть элементы из $A$, которые нужно найти. Элемент $x \in A$ 
представляется в виде линейной комбинации мономов, и найти $x-$ значит найти коэффициенты при них.

Как и вьше, каждой вершине $v$ дерева $T$ отвечает моном $M(v)$. Кроме того, вершине $v$ отвечает набор коэффициентов при мономе $M(v)$ для каждого $\left\{x_{i}\right\}_{i=1}^{k}$ и для $g-$ всего $k+1$ коэффициент, а значит и $(k+1)$-мерное векторное пространство $V(v)$. Ясно, что равенству (2) отвечает набор соотношений на коэффициенты:

$$
\sum_{M \in T ; i=1}^{k} \lambda_{f_{i}}(N, M) \delta\left(M, x_{i}\right)=\delta(N, g), \quad N \in T
$$

Легко видеть, что условия теоремы 1 удовлетворяются.

Аналогичным образом, в п.в) надо проверить неразрешимость уравнения

$$
\sum_{i=1}^{k} x_{i} f_{i}=0
$$

Вершине $v$ дерева $T$ отвечает набор коэффициентов при мономе $M(v)$ при $\left\{x_{i}\right\}_{i=1}^{k}$. Такие наборы образуют $k$-мерное векторное пространство $V(v)$. СЛР получается путем приравнивания коэффициентов в равенстве (4):

$$
\sum_{M \in T ; i=1}^{k} \lambda_{f_{i}}(N, M) \delta\left(M, x_{i}\right)=0, \quad N \in T .
$$

Частньм случаем автоматной мономиальной алгебры является конечно определенная алгебра, в частности, свободная конечно порожденная алгебра. Предыдущее следствие можно обобщить.

ОПРЕДЕЛЕНИЕ. Пусть на мономах алгебры $A$ с фиксированной системой образующих задан порядок, для которого существует нормальны й базис, т.е. базис из мономов, не представимых в виде линейной комбинации меньших. Алгебра $A$ назьвается алгеброй с ограниченной переработкой справа, если при некотором $d \in \mathbb{N}$ для любого нормального слова $W$ в алгебре $A$ и любой образующей $a_{i}$ выполняется равенство

$$
W a_{i}=\bar{W} \cdot\left(\sum_{j} \lambda_{i j} W_{j}\right)
$$

где $\lambda_{j} \in \mathbb{F}$ для всех $j$, причем $W W_{j}$ суть нормальные слова алгебры $A,\left|W_{j}\right| \leqslant 2 d, \mathrm{a}$ слово $\bar{W}$ есть начальный кусок слова $W$ длины $|W|-d$.

Если множество слов нормального базиса образует регулярньй язьк, каждому слову отвечает вершина автомата и все коэффициенты $\lambda_{i j}$ зависят только от типа вершины графа, отвечающего слову $W$, то $A$ есть автоматная алгебра с ограниченной переработкой справа.

Аналогичным образом определяется (автоматная) алгебра с ограниченной переработкой слева. 
ЗАмЕчАниЕ. Если порядок на словах нетеров и согласован с умножением, то условие $\left|W_{j}\right| \leqslant 2 d$ излишне (поскольку всегда $W_{j} \leqslant b a_{i}$, где $W=\bar{W} b$, и таких $W_{j}$ конечное число, так что это условие для некоторой константы $d$ будет выполняться).

Смысл этого определения заключается в том, что умножение на образующую не сильно меняет нормальную форму.

Если множество определяющих соотношений алгебры $A$ с ограниченной переработкой образует конечньй базис Грёбнера, то $A$ автоматна.

ЗАмечаниЕ. Пусть $A$ - алгебра с ограниченной переработкой справа, $D$ - конечное множество слов. Тогда для всех $u \in D$ имеет место равенство

$$
W u=\bar{W} \cdot\left(\sum_{j} \lambda_{j} W_{j}\right)
$$

где $\lambda_{j} \in \mathbb{F}$ для всех $j$, причем $\bar{W} W_{j}$ суть нормальные слова алгебры $A,\left|W_{j}\right| \leqslant 2 d^{\prime}$, а слово $\bar{W}$ есть начальньй кусок слова $W$ длины $|W|-d^{\prime}$. Константа $d^{\prime}$ зависит от набора $D$ (и алгебры $A$ ).

Если $A$ автоматна, то все коэффициенты в равенстве (6) зависят только от слова $u$ и типа слова $W$ (т.е. вершины графа Уфнаровского).

Любая мономиальная алгебра есть алгебра с ограниченной переработкой (слева, а также справа) и не обязательно конечно определена.

Пусть $A$ - автоматная алгебра с ограниченной переработкой справа (соответственно, слева). Тогда множество определяюших соотношений в $A$ имеет вид

$$
W u_{i}=\sum_{i j} \lambda_{i j} \bar{W} u_{i j}
$$

где $\lambda_{i j}$ зависят только от типа слова $W$. Тем самым оно задается конечным набором данных.

Приведем еще одно следствие из основной теоремы.

СлЕДСТВИЕ 2. Пусть $A$ - автоматная алгебра с ограниченной переработкой справа (соответственно, слева), константа $d$ и базис Грёбнера для множсества определяющих соотношений алгебры $A$ заданы. Тогда $A$ задается конечным числом данных и существуют алгоритмы проверки следующих утверждений:

а) данный әлемент $x \in A$ (т.е. линейная комбинация слов) является правым (соответственно, левым) делителем нуля;

б) данныи әлемент $g \in A$ принадлежит левому (соответственно, правому) идеалу, заданному конечным набором әлементов;

в) алгоритмически разрешим также вопрос о том, является ли правый (соответственно, левый) идеал свободным А-модулем с данной конечной системой порождающих. 
ДокАЗАТЕЛьСТво. Поскольку множество слов автоматно, для дерева $T$ и системы векторных пространств конструкции те же, что и в доказательстве предыдущего следствия, вершине дерева отвечает моном, моному отвечает набор коэффициентов, с которыми он входит в элементы $A$, которые надо найти, и в правые части уравнений. Задачеп.б) отвечает уравнение

$$
\sum_{i=1}^{k} x_{i} f_{i}=g
$$

задаче п.в) - уравнение

$$
\sum_{i=1}^{k} x_{i} f_{i}=0
$$

СЛР строится в точности как и в предыдущем следствии, надо лишь видоизменить определение величины $\lambda_{f}(N, M)$. В равенствах (8) и (9) возникающиепроизведения пар нормальных слов следует переписать в виде линейных комбинаций нормальных слов и рассмотреть возникающие коэффициенты при нормальных словах. Поэтому $\lambda_{f}(N, M)$ есть коэффищиент при $N$, получающийся, если произведение $M f$ расписать в виде линейной комбинации нормальных слов. Уравнения на коэффициенты будут иметь в точности такой же вид, что и равенства (3) и (5).

В силу замечания после определения алгебр с ограниченной переработкой, эти коэффициенты $\lambda_{f_{i}}(N, M)$ зависят только от типов соответствующих слов и от пути, их соединяющего. Кроме того, коэффициент при мономе $M$ зависит только от тех коэффициентов при мономах, входящих в $\left\{x_{i}\right\}_{i=1}^{k}, g$, которые отвечают вершинам дерева $T$, находящимся на расстоянии не больше чем $2 d^{\prime}$ от вершины дерева $T$, отвечающей $M$; таким образом, выполняются условия локальности (константа $d^{\prime}$ зависит от множества ненулевых мономов, входящих в $f$, и от $A$ ). Поэтому можно применить теорему 1.

ЗАМЕчАнИЕ 1. Условие следствия вьполняется для алгебр с ограниченной переработкой справа в нашем смысле, если множество определяющих соотношений образует конечньй базис Грёбнера (фактически это доказано в работе [6]). Это алгебры с ограниченной $R$-переработкой в смысле [6]. Они рассматривались в работах [3], [5], [6]. Данное следствие обобщает как случай автоматной мономиальной алгебры, так и случай алгебр с ограниченной переработкой в смысле упомянутых работ.

ЗАмЕчАниЕ 2. Интересно построить для алгебр с ограниченной переработкой теорию, аналогичную теории мономиальных алгебр, - в частности, доказать совпадение ниль-радикала и радикала Джекобсона. На автоматные алгебры с ограниченной переработкой, по всей видимости, непосредственно переносится теория из [3], [5], [6]. Наше определение алгебр с ограниченной переработкой несколько отличается от определений из этих работ (там старший член нормальной формы $u a_{i}$ зависит только от конца ограниченной длины слова $u$ ). В нашем случае любая мономиальная алгебра имеет ограниченную переработку (обнуляющее влияние начала возможно), а случай автоматных алгебр с ограниченной переработкой обобщает случай алгебр с ограниченной переработкой (в смысле [3], [5], [6]), имеющих конечньй базис Грёбнера определяющих соотношений. 
ЗАмЕчАниЕ 3. Алгоритм, получаюшийся из доказательства основной теоремы, можно улучшить с помощью такого соображения. Назовем два вектора фрагмента әквивалентными, если замена одного другим сохраняет вьполнимость уравнений, отвечаюших вышележашим вершинам. Классы эквивалентности могут образовьвать пространство существенно более низкой размерности.

ЗАмЕчАниЕ 4. Проблема нахождения алгоритма проверки, является ли элемент данной мономиальной алгебры делителем нуля, родилась из более общего вопроса, поставленного В.Н. Латьшевьм. Пусть $A$ - конечно порожденная и конечно определенная алгебра с конечным базисом Грёбнера для идеала определяющих соотношений. Сушествует ли алгоритм проверки того, является ли данньй элемент делителем нуля (нильпотентом)? Предполагаемьй ответ отрицателен.

\section{СПИСОК ЦИТИРОВАННОЙ ЛИТЕРАТУРЫ}

[1] Bergman G. The diamond lemma for ring theory // Adv. Math. 1978. V. 29. № 2. P. 178-218.

[2] Белов А.Я., Борисенко В. В., Л атышев В.Н. Мономиальные алгебры // Итоги науки и техн. Совр. матем. прил. Тем. обзоры. Алгебра-4. Т. 26. М.: ВИНИТИ, 2002. С. 35-214.

[3] Иыуду Н.К. Алгоритмическая разрешимость проблемы распознавания делителей нуля в одном классе алгебр // Фундамент. и прикл. матем. 1995. Т. 2. № 1. С. 541-544.

[4] Иыуду Н. К. Стандартные базисы и распознаваемость свойств алгебр, заданных копредставлением // Дисс. ... к.ф.-м.н. М., 1996.

[5] Пионтковский Д. И. Базис Грёбнера и когерентность мономиальной ассоциативной алгебры // Фундамент. и прикл. матем. 1996. Т. 2. № 2. С. 501-509.

[6] Пионтковский Д. И. Некоммутативные базисы Грёбнера, когерентность ассоциативных алгебр и делимость в полугруппах // Фундамент. и прикл. матем. 2001. Т. 7. № 2. С. 495-513.

[7] Уфнаровский В. А. Комбинаторные и асимптотические методы в алгебре // Итоги науки и техн. Соврем. пробл. матем. Фундамент. направления. Т. 57. М.: ВИНИТИ, 1990. С. 5-177.

[8] Воробьев К. Возвратные последовательности. М.: Наука, 1970.

Московский институт открытого образования

Поступило

Hebrew University of Jerusalaem

22.05 .2003

Исправленный вариант

09.03.2005 